\title{
CONSERVATISM IN BRIDGE-WORK
}

\author{
By FRED B. HART, D.D.S., San Francisco, California
}

(Read before the Chicago Dental Society, Fcbruary 21, 1922)

$\mathrm{T}$ $\mathrm{HE}$ problem confronting the dentist to determine the best means of supplying missing teeth has always carried many perplexities and responsibilities; but the past few years our literature has been so filled with conflicting opinions that the scrupulous and conscientious operator has often been influenced to the point of experiencing considerable difficulty to decide how best to serve his patient.

The fixed bridge has been the object of severe attack since Hunter in 1911 called attention to the menace to health resulting from the insanitary condition of mouths due to our much-boasted American dentistry, and the methods generally in vogue received a blow a short time afterward by Rosenow's indictment of the apical abscess.

The accusation made by Hunter aroused indignation and resentment at first, but it was closely followed by an awakening that structures which we had realized long before were not monuments to the most wholesome cleanliness, must, because of the general acceptance of the responsibility for systemic disorder, undergo a change.

Mention was made that Rosenow's charge affected all procedures then in vogue. We must not be unmindful that Dr. Peeso had long before recognized the desirability of eliminating unhygienic influence of the fixed bridge, and made bridges that permitted removal, but his procedure, which required the loss of the pulp, as well as those who favored the fixed bridge and advocated its removal was brought under the ban by the possibility of the pulpless tooth to abscess.

Much has been done in both the fixed and the removable bridge to overcome these objectionable features. We must credit the work of Dr. Tinker and others in putting the fixed bridge on a higher plane, and it is also to the credit of these men that they have been fair enough to acknowledge that the fixed bridge has limitations and to look with favor upon a removable structure, could one be supplied that would give lasting and satisfactory service.

Some of the supporters of the removable bridge have not been so liberal in their views, as they have deprecated in every particular the employment of the fixed bridge, and have mercilessly attacked the ideals of those who advocate its use, even criticizing colleges for continuing to teach it. Such drastic condemnation has been given much prominence in certain of our publications, and while some good may have come from it, it seems to me that the cause would be better served by a more lenient attitude.

Webster gives as a definition of a conservative, "One who respects old institutions; not subject to change simply for the sake of change." It is my desire to 
qualify to that definition. I am going to submit some ideas that probably cannot be considered among the older methods, but $I$ respect the old institutions and have not changed simply for the sake of change.

Although interested in the development of removable bridge-work, and with great confidence in the advisability of its use, I could not get pleasure in entirely condemning the fixed bridge, if for no other reason than that $I$ meet among my friends every day someone who tells me of a bridge that gave pleasure and profit to the patient, and the belief that such cases demand a place as successes. We respect the opinions of many of the men who cite such cases; we credit their ability to observe, and esteem their truthfulness. I feel no greater affliction could be had than fcr one to become so obsessed with his own ideas as to stunt the appreciation for his fellow-worker, to disrespect his honesty of purpose, and to discredit his ideals.

As before mentioned, however, most zealous fixed bridge supporters admit the advantage of having a structure that can be removed for cleaning, and he may also acknowledge that many of the requirements of construclion for the fixed bridge are undesirable. From the fact that most operators prefer when possible, to construct a bridge with one end given some freedom of movement, it is an acknowledgment that it is desirable not to tie two teeth rigidly together if it can be avoided. It is also considered, where two teeth are so tied together, that the abutment restorations must be very strongly made and must firmly grasp the tooth. To do this often means excessive cutting and the necessity of resorting to such restorations that impair and require much of the natural contour of the tooth to be restored.

The fact that the abutments must be placed in parallel relation when both ends of the bridge are fixed, together with the rigid holding of the teeth, limits the use of the inlay which would disturb the smallest amount of natural tooth contour when used as abutment restoration.

In cases of strongly converging or diverging teeth great difficulty is often experienced in bringing about the parallelism required by fixed structures without pulp devitalization. All these are factors to influence a conclusion that a large percentage of cases would be better cared for by a removable structure if we have ore that does not offer objectionable features that would overbalance those attributed to tha fixed type.

It is not surprising that in the endeavor to perfect or to originate ideas for the correcting of some fault, extravagant claims might result from the concentration of thought exerted over an extended period of time in that effort. Both the dental and the medical professions have keen swayed by this influence. We can all recall an endless number of instances in which the profession has been so affected, and bridge-work has probably come in for more than its share in recent years. An example is given in the socalled movable-removable bridge. While it has been conceded by many thoughtful dentists that it is not best to rigidly tie healthy teeth together, and that it is desirable to have some yield permitted as the force of mastication is applied on the individual abutment tooth, or the artificial teeth supplied, very extravagant claims have been made and have had much influence.

It is believed that the latero-rotary movement of the movable-removable bridge which has received so much mention and has been credited with such beneficial effects upon the mucous membrane and all subjacent tissues, has been accomplished in many cases as a detriment to the attachment to the abutment teeth. A bridge that has been constructed 
with attachments that are not placed in parallel relation to each other buccolingually cannot rock as described by the originator without exerting a leverage upon the abutments, yet the tendency to yield is increased because they can seldom occupy a position in line with the stress of occlusion.

It is claimed that the mucosa takes the stress, and that the attachment acts only as a guide which, if true, would still exert a leverage by its movements, but it is also asserted that a check is made by the attachment before the limit of elasticity of the mucosa is reached, and if that is so, damaging results may be expected when it occurs.

The most destructive factor we have to contend with in all our work is the force applied by the teeth in masticating. No bridge, fixed or removable, can be other than damaging if the occlusion is not properly centralized, and we consider that the removable bridge should certainly have its attachments placed in line with that stress. We feel that the first thing in constructing a bridge, even before the decision is made as to what type of structure is to be used, should be a careful study of the direction of the force as would be applied in mastication.

A large attachment, which must be placed in a tooth as it will best receive it and will not permit the adjustment necessary to compensate for this important factor, cannot safely be used. Many ideas of the so-called movable-removable bridge seems to be founded without sufficient regard for these mechanical principles. Consider the arching of attachments so they will more readily yield a rocking motion, spoken of as a rhythmic movement. To arch to a central position on an attachment will not help matters if that point is not in line with the stress as applied. It has been said that if the attachments are not arched they will arch themselves. If this is a fact the force of occlusion is responsible for it, and the farther that is applied from the central position on the attachment the greater is that influence, and we have a movement, the degree of which cannot be estimated, and wear is checked only as the force is spent.

Many other claims have been made that are not well founded upon safe mechanical principles. Some of these are being discontinued, which shows the indiscretion of so harshly criticizing the work of others.

The term "removable bridge" has been stretched to cover all replacements where some teeth are still remaining in the arch, when in reality perhaps all removable bridges should be considered partial dentures, or strictly speaking, are partial dentures. I wish, however, in referring to removable bridges, to be understood as considering them in positions that have formerly been looked upon as positions for fixed structures.

Many systems and means of attachment have been advanced, all of which have merit, and all, perhaps, have their limitations. The system that has been evolved and which I shall now submit to you may well be included in that class. No claim is made for it as being a panacea; in fact, it is felt that much injury often results from such extensive claims, as some are apt to try to use it where failure could be the only result. What the profession probably needs is a thorough understanding of the various systems, and then a greater development as engineers to know when and how to proceed.

The thought first suggesting the inception of this system was that it was not ideal to select a given attachment because it was manufactured to be used in a certain position. A smaller attachment was needed, and yet one that was strong and offered sufficient surface for frictional resistance against dislodgment. The thought of being able to place the 
attachment in line with the stress of occlusion became paramount, and to that end a two-piece stud or male portion was evolved that would permit the combinations to be formed which would offer the advantages of placement in the tooth where it would best support, and also guard against disturbing the normal tooth contour or encroaching upon the pulp. Another consideration was that the best retentive form of cavity, if an inlay was to be used as an abutment restoration, was one that permitted parallel perpendicular walls with sufficient tooth structure remaining to insure strength. That the adaption might be made to fit the condition, further grinding or shaping of the stud may be done by the dentist, after which the socket is formed around it by an instrument devised for that purpose. Thus an attachment is made to meet the individual needs of the case.

That it is necessary to parallel the sockets so the bridge may be removed is realized, and to do so mandrels are usually made to fit the body of the sockets, but as the privilege has been afforded to make different combinations and to further shape attachments to suit the case, mandrels that would fit that portion of the socket would not be practicable. The slot in the socket which adapts to the stem of the stud is of unvarying width, and mandrels are made to accurately fit into it, which are held securely by means of a screw, the head of which projects over the slot and gauges the material.

A paralleling instrument of small and simple design is used to secure vertical parallelism.

To avoid the possibility of having the attachments stand in relation to each other that would not permit centralizing in line with the stress of occlusion, pins are inserted in the hubs of the mandrels and a side paralleling instrument is used which insures us that the stems of the stubs are in a parallel relation to each other. While the sockets are being soldered in their respective abutments, carbons which are shaped to fit the slot of the socket are used to hold them in position during this operation, and the construction of the bridge proceeds.

With these attachments the following accomplishments are attainable; the inlay can well be used for an abutment restoration which is no doubt the most ideal form we have today for that purpose; more accurate tooth form can be maintained; excessive cutting of the tooth, either of the periphery or toward the pulp, is unnecessary. Outline form and best retentive form of cavity can be accomplished. Greatest strength is afforded, as the attachment takes stress not only across the ends of the bucco-lingual portion of the stud but on the stem close to the bridge unit. The claim is not made that the mucous membrane can be asked to take more than a small amount of the stress applied in mastication. The great advantage claimed is the possibility for adapting in line with the stress of occlusicn.

They are retained against removal by the sockets being adapted over each individual stud, and not only fitting around the body of it but tightly fitting on each side of the stem, while slight spreading of stud will compensate for wear.

Inasmuch as we are considering this subject from a conservative standpoint we feel justified in mentioning briefly the cconomic side of the bridge-work situation. Some have denounced the fixed bridge to the extent that they would deny its employment even if removable bridges were beyond the financial possibilities of the patient.

We are going ahead, but we should bear in mind that we must still look care- 
fully on all sides to see that our theories and principles are such as will stand the test, and will assist in taking a place as a permanent and satisfactory service.

\section{Discussion}

M. G. Kral: Ever since removable bridgework made its appearance considerable agitation has taken place in regard to its use. On the one hand were its supporters. On the other, those who ridiculed and condemned it. Fixed bridge-work seemed to have so many disadvantages and to be so inadequate in many cases that we decided to try a few experimental restorations of the removable kind. As these were rather extensive cases of long spans naturally we were skeptical as to the final outcome. But as these cases came before us for check-up examination from time to time and the conditions noted, we were amazed at the wonderful results. Consequently the experiments were extended, bridges of shorter spans constructed with equally gratifying results.

The essayist has given us his idcas of conservatism in bridge-work and by so doing continues to leave the burden of decision upon the individual operator whether a fixed or removable structure should be employed in the restoration of missing teeth. After observing hundreds of cases my question is, if a removable structure is advisable in certain specific cases, why not wherever any bridge is indicated? Must we go on giving those who place themselves in our care unscientific and unhygienic restorations?

Let us give credit to those who have fostered improvements along any lines of bridge-work, but on the other hand let us condemn the practices which have produced pathologic mouth conditions. I protest against the use of the gold shell crown, open face or slipper crown, the banded Richmond crown and any or all restorations which cannot be made to conform to the contour of tooth surfaces. What good can be derived by the removal of infected teeth if we replace them with artificial substitu'es whose abutments produce lesions in the investing tissues equally dangerous to the health of the patient.

The scope of our responsibility to the patient has a very wide range. Our work has not been well done, providing nothing has been accomplished aside from the construction of the appliances. The mere employment of special attachments cannot insure the success of a case unless all of the countless details are worked out in their entirety.

Naturally our ideal is a finished piece of work which when placed in the mouth will please the patient both as to comfort and appearance. We must know that we have conserved the pulps of our abutment teeth inasmuch as we have employed a conservative plan of tooth preparation and that our patients can and do get the maximum amount of usefulness and service from their bridge-work.

Prior to planning our case we must take into consideration the conditions of the mouth:

1. Accurate models from plaster impressions of mouth for reference and laboratory study.

2. A full mouth radiograph of all teeth and supporting structures.

3. Removal of all abscessed or granulomatous teeth, those having lost considerable of the supporting alveolus and peridental membrane, and all broken off roots. Ample time should be given for the absorption of alveolar process be ore placing restorations of a removable nature.

4. Removal of all deposits of calculus. Corrections should be made on all teeth bearing imperfect fillings and crowns. Restore contacts and natural tooth contours. Nature provides too'h contours for the protection of the surrounding soft tissues; therefore every line curvature and prominence are there for a definite purpose. Whatever that may be it is our duty to know and to reproduce them.

5. Study the cusp interdigitation. Note and correct the high points in the lateral excurs on of the mandible. We must bear in mind that this lateral movement takes place hundreds of times during each meal. The investing structures or tissues are not rigid, therefore any point or cusp of a tooth occluding before any great pressure has been exerted will in time loosen the tooth.

6. Visualizic the finished work. Have a thorough knowledge of the method to be pursued, get a mental picture of the case as it should be finished and lay plans accordingly.

Inasmuch as $\mathrm{I}$ believe the best results are attainable only by the use of movable-removable bridge-work, carefully planned and constructed, a few suggestions at this point may not be out of place since practically every dental school has seen fit not to include this technic in the curriculum of the regular school work, making it necessary for the dentist to look about for o'her means of mastering th 3 subject.

Perhaps the most logical method to pursuc is by planning and actually constructing a number of dummy cases on a typodont, as it is hardly fair to experiment on practical cases until a fair amount of technic is gained. Having mastered that, select a simple practical case of a single missing tooth in the posterior of the mouth. As success and confidence is gained one may take on more complex and intricate cases.

Before we go farther let us consider removable bridge attachments. The consensus of opinion among modern bridge-workers is that 
the bucco-lingual type of attachment is the most desirable.

An attachment must be of such size that will permit soldering into inlays without excessive extension of cavities or the over-contouring of inlay.

It must be interchangeable which calls for uniformity, precision and accuracy in its manufacture. I disagree with the essayist that this can be done by hand.

The design must be for frictional retention rather than wedging retention. An attachment must be so planned and constructed that it will permit of individual tooth movement.

The point trought out by the essayist for a lateral parallelism as well as the perpendicular is good.

\section{CONSTRUCTION OF CASE}

Select a simple tridge, one where the artificial substitute and the abutments are subjected to the same physical stress, that is, where the stress is in the same direction.

The G. V. Black rules of cavity preparation are carried out in all details.

Having cut cavities put matrix on tooth, soften wax to proper plasticity and force in one direction, hold in position until it stops yielding, remove to see that all points are reproduced, remove band and trim to anatomical form, finish all surfaces leaving occiusal to be finished last.

Approximately trin out a seating for the bucco-lingual jacket.

Remove, invest anl cast. Use a hard gold alloy for casting. Place castings in cavities; test occlusion.

Reduce soft tissues with a one-thousandth per cent solution adrenalin chloride. The impression is then taken in plaster. It is best to remove impression and inlays intact where possible.

Inlays should be seated securely and abutment walled off carefully. Pour low fusing metal into this form. Allow metal to chill, remove moldine. Impression is varnished in the usual manner and artificial stone is packed into the remaining part of impression. Allow time for setting. Separate. Be certain that the inlay is perfectly seated in the cavity and that there is sufficient room for housing the bucco-lingual jacket in all directions. It is desirable to place attachments at a $5^{\circ}$ angle mesially. Parallel attachments carefully and wax in position. Carbons anti-fluxed are placed in slots; cavo-surface also anti-fluxed. Invest in refractory compound. Boil out wax with stream of running water. Remove surplus investment, flux care ully and solder. Clean carefully in hydrochloric acid and place in cavities. Test for parallelism before trimming occlusion.

Adjust slots to accommodate male part as quite often in soldering the contraction of solder has caused an irregularity in the buccolingual jacket; sizing tools of proper dimensions are indicated for this step. Burs, files or sharp instruments must never be used within the bucco-lingual jacket.

Teeth of proper size and shade are selected. It is often necessary to correct or modify a shade or contour by the addition of porcelain. In specific cases entire teeth are built up much like jacket crowns.

Cut teeth to fit model before carving occlusal surface. Grooves are then cut on mesial and distal surfaces for trusses which are to receive the stem of male portion of attachment. Prepare teeth so there is an even distribution of wax thruout. This does more to insure satisfactory casting than all other precautions.

Conform two layers No."28 gauge wax to model. Press out wax pattern over tooth, trim down to one thickness No. 28 gauge wax over portion to be attached to saddle, place in position, tack and contour with molten casting wax; trim to desired size, remove and cast.

In reference to cas:ings, never use anything but the most reliable golds made especially for removable bridge-work.

After casting is madc, examine for nodules, removing any if present. Attempt to seat tooth. If it binds do not force it. Remove each time it binds, relieve offending spot until tooth finally reaches position.

Trim and polish with too $h$ in position, always against tooth to prevent distortion of margins.

Cut slots in trusses with fissure burs and separating discs. Wax stem of attachment to truss. Remove and solder one at a time. Cut off excess stem. Reseat teeth and refinish. Bridge is then ready for trial in mouth, where final adjustments are to be made.

G. Walter Dittmar, Chicago, Illinois: I should like to call attention to one or two things in connection with the paper. The first is the method of making the stud to fit the cavity. In other words, he has a process by which he can reduce it to a size and form that will fit the particular cavity. If he has a tooth, the cavity of which is small, he can make a stud and then make the sleeve fit accurately over the stud which is to be inserted into the inlay. The little device for making these sleeves is wonderfully simple and accuraie. The parallelometer devised is so simple and yet so accurate that $I$ think it is worth while giving it special mention.

I still believe there is a decided field for fixed bridge-work, and that there always will be until we get to that particular position where we do not need bridges. I think the fixed bridge has its place. However, it is not intended for all cases, nor are removable bridges suitable for all cases.

Removable bridge-work from the standpoint 
of being more sanitary has a decided advantage. If you can take a bridge out, scrub it, and put it back, it is cleaner than you could get it with a toothbrush, if fixed in the mouth. However, a fixed bridge placed where it is indicated can be kept as clean as the natural teeth if the patient is taught how and makes an effort. I do not think that many people are poisoned by the accumulation on their own fixed bridges. Individuals become immune to a cetrain extent. I do not wish to be understood as decrying the dangers to focal infection, or favoring filthy mouth conditions, but it is a well known fact that patients establish a certain amount of immunity to such conditions.

Dr. Hart is right in saying that "the more immovable a bridge is, the more efficient it will be for masticating purposes." The more movable it is, the more it gives, and consequently will not enable one to masticate the food as thoroughly. From that standpoint, a fixed bridge is better than most removable ones.

The criticism was made that this movableremovable system was not being taught in our dental schools. The method is too complicated to teach in dental schools. I believe the reason the average prosthetic teacher is not teaching this particular system is because ne is not as yet convinced that it is fundamentally right. The method is too young. The fact that it is ten or fifteen years old does not mean very much. The fact that some of these removable bridges have been successful for four or five years or even ten years does not mean much. I have seen fixed bridges that have been worn for twenty and thirty years and are still doing excellent service. After this process has been in use by the profession for thirty years or more, we can then look back and see whether it is successful or not.

Hart J. Goslee, Chicago, Illinois: This subject of "removable" bridge-work versus "fixed" bridge-work, as it is being presented to the thinking members of the profession today, causes some of us to pause and study the statements that are being made by the advocates of these respective methods.

The only value of any method lies mainly in its range of application. And I question very seriously whether the range of application of that particular type of removable bridge-work, which is being advocated by the essayist is not very much more limited than they would have us think.

There is a range of application for all of the various types of attachments for removable bridge-work, and there is a very great range of application and usefulness for all forms of removable fixtures, and yet it is my honest and candid conviction that there is also a great range of application and of usefulness for fixed structures. One method is indi- cated in one place, and another in another place, and in proportion as the bridge by which you supply missing teeth is movable, in that same proportion is its efficiency for masticatory purposes minimized or reduced; just in proportion as you make your structure fixed, in that same proportion is its efficiency for masticatory purposes increased. However, I would not have you think from what I have just said that every dental bridge should be a fixed bridge.

All of us have learned a very great and most important lesson and that is that we erred greatly in the past by making too many large structures of a "fixed type." But there is and I think always will be many places for a fixed bridge, where but two or three teeth of similar functional activity are involved. 'The increased efficiency which can obtain by supplying missing teeth in a fixed manner, will always warrant us, in my judgment, wherever the conditions are favorable, to give preference even to "fixed" bridges, providing that we will always remember that sanitation is one of the requirements which must never be overlooked.

It is a somewhat singular thing that these removable bridge attachments are shown only as applied to small structures, involving but two attachments and two missing teeth, the very best place for a fixed bridge.

Dr. Ottolengui, who is a disciple of $\mathrm{Dr}$. Chayes, has been discussing the question of movable and removable bridge-work for many years, but we do not agree. In my office once he removed from his lower jaw a beautiful piece of workmanship which involved the right first molar in a removable bridge and said, "That is the only way missing teeth should ever be supplied." The workmanship was beautiful, but I do not know how anyone ever got those two inlays in without devitalizing the pulps. That bridge was not any more sanitary than a fixed bridge would have becn. I advised him to cut off that saddle, and cut down the approximal sides of that dummy, and cement it in, and that he would have a more useful structure.

Almost all of these removable bridges where that type of attachment is used are simple, small fixtures. They did not show us bilateral structures involving both sides of the arch and some of the anterior teeth. Those are the cases where removable bridges are indicated, and where you have to use them. It is not a question of whether you want to or not or whether you believe in them or not. Where we are to supply missing teeth on both sides of the arch, with the possibility also of missing anterior teeth, one or more, we must have a removable structure, and a single structure in those cases is better than several small ones. I never make a fixed bridge of more than three or four teeth, and I prophesy that we shall always do fixed bridge-work to that extent. 
When a bridge is larger than that, then a removable one is demanded.

I do not believe very much in the theory of the massaging of the soft tissues by the saddles in removable bridge-work. Certainly, the soft tissues of the mouth will yield more readily to pressure than will the supporting teeth, but the soft tissues cannot yield until the supporting teeth likewise yield and therefore any possible advantage in the way of the massaging of these tissues by the slight mobility of the fixture is nil. However, we do not have the same degree of stasis of capillary circulation because there is no definite pressure at one localized point all the time; therefore, the circulation in these tissues will remain more normal than if the structure was absolutely immovable and perhaps slightly imbedded, and to that extent we would have a more normal condition. But the condition is not made more normal by the slight mobility of the fixture. It is more normal because there is no pressure to interfere with capillary circulation.

In conclusion, I wish to say that there is a place for this type of attachment, and the suggestions offered by Dr. Hart will enable us to obtain the advantages to which I have referred. But I have found that all methods requiring the same degree of exactness are beyond the possibility of the average dentist, either for reasons of money, of time, or of ability, and therefore these methods will always to a greater or less extent be laboratory methods. The average dentist cannot or will not do it, and therefore the work will be delegated to the dental laboratory, and it is to the dental laboratory man of high skill and experience that we will have to look for the successiul application of these various methods. There are more simple methods which we can use successfully, because we can do more injury to good sound teeth by utilizing a method which we cannot apply with skill than we will do to good sound teeth by using any method, however simple, that we may be able to skilfully master.
Nathan M. Kaplan, Chicago, Illinois: Dr. Hart has given us a useful system and a new idea. In making these boxes so close to the attachment, using a stud as a guide, if the box is soldered to the inlay, the box will be so tight that it will be impossible to insert the stud and take it out with ease. Dr. Hart claims that the box must be trimmed and made to fit. These types of attachment must fit the box in its entirety, not at one point like a wedge, depending on personal contact. I can see no difference in preparing the cavity and making the attachments to fit the cavity, or in manufacturing the attachments so small that it can be made useful and applicable in all cases. $I$ believe this system is necessary on account of the fact that most of the attachments on the market are too large, and that smaller attachments are required for this type of work. I want to bring out the point that by manufacturing the attachment in accordance with our own ideas we get a far better attachment than by pursuing this particular method.

Dr. Hart (closing): The advantage of removable bridges is that they can be taken out, cleaned, and put back again. Patients have every opportunity for removing these bridges and of keeping them clean.

In taking a conservative attitude in regard to bridge-work, I feel that fixed bridge-work can be successfully used and it has its place. On the other hand, there are many conditions in which small removable bridges are indicated. In other cases larger removable bridges can be used successfully.

It has been stated in the discussion that the average dentist cannot put in removable bridgework skilfully and successfully. I have been talking along lines that the average dentist can aspire to. We want the average dentist to raise the standard and to do the very best that can be done in dentistry. I cannot see how dentistry is going to make much progress if we are going to make the standard fit the lower grade of men, considering the average is as low as was stated in the discussion. 